\title{
A bimodular PKS platform that expands the biological design space
}

Amin Zargar, ${ }_{1,2,4}$ Luis Valencia $^{1,2,6}$, Jessica Wang ${ }^{1,2}$, Ravi Lal $^{1,2}$, Samantha Chang ${ }^{1,2}$, Miranda Werts $^{1,2}$,

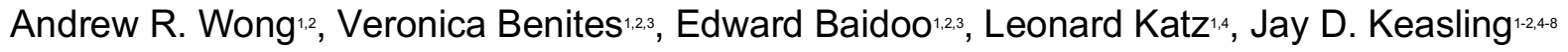

1 Joint BioEnergy Institute, Lawrence Berkeley National Laboratory,

Emeryville, CA 94608, United States

${ }^{2}$ Biological Systems and Engineering, Lawrence Berkeley National Laboratory, Berkeley, California, USA

${ }^{3}$ Department of Energy Agile BioFoundry, Emeryville, CA 94608, USA

${ }^{4}$ QB3 Institute, University of California-Berkeley, 5885 Hollis Street, 4th Floor, Emeryville, CA 94608, United States

${ }^{5}$ Department of Chemical \& Biomolecular Engineering, University of California, Berkeley, CA 94720, United States

${ }^{6}$ Department of Bioengineering, University of California, Berkeley, CA 94720, United States

${ }^{7}$ Novo Nordisk Foundation Center for Biosustainability, Technical University

Denmark, DK2970-Horsholm, Denmark

${ }^{8}$ Synthetic Biochemistry Center, Institute for Synthetic Biology, Shenzhen Institutes for Advanced Technologies, Shenzhen, China

${ }^{*}$ Corresponding author

Jay D. Keasling

jdkeasling@lbl.gov 


\section{Abstract}

Traditionally engineered to produce novel bioactive molecules, Type I modular polyketide synthases (PKSs) could be engineered as a new biosynthetic platform for the production of de novo fuels, commodity chemicals, and specialty chemicals. Previously, our investigations manipulated the first module of the lipomycin PKS to produce short chain ketones, 3-hydroxy acids, and saturated, branched carboxylic acids. Building upon this work, we have expanded to multi-modular systems by engineering the first two modules of lipomycin to generate unnatural polyketides as potential biofuels and specialty chemicals in Streptomyces albus. First, we produce $20.6 \mathrm{mg} / \mathrm{L}$ of the ethyl ketone, 4,6 dimethylheptanone through a reductive loop exchange in LipPKS1 and a ketoreductase knockouts in LipPKS2. We then show that an AT swap in LipPKS1 and a reductive loop exchange in LipPKS2 can produce the potential fragrance 3-isopropyl-6-methyltetrahydropyranone. Highlighting the challenge of maintaining product fidelity, in both bimodular systems we observed side products from premature hydrolysis in the engineered first module and stalled dehydration in reductive loop exchanges. Collectively, our work expands the biological design space and moves the field closer to the production of "designer" biomolecules.

\section{Highlights}

- Engineered lipomycin module 1 and module 2 to produce unnatural polyketides as valuable bio-based chemicals

- A reductive loop swap and ketoreductase knockout used to produce $20 \mathrm{mg} / \mathrm{mL}$ of a novel ethyl ketone, a gasoline replacement

- An acyltransferase swap and reductive loop swap successfully produced $\delta$-lactone, a potential fragrant compound

- Incomplete reduction and premature hydrolysis observed in engineered modules 


\section{Keywords}

PKS; biofuels; reductive loop exchanges; $\delta$-lactones; ketones

\section{Graphical abstract}

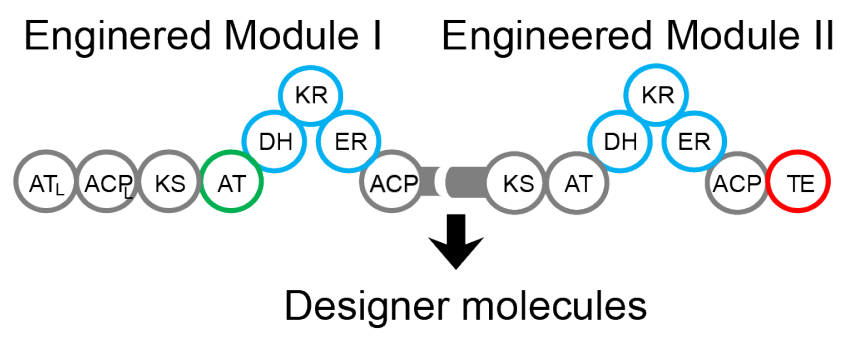

\section{Introduction}

Advances in biotechnology have only begun to capitalize on the biomolecular design space. Referred to as the parvome, 'parv-' meaning small and '-ome' denoting group, the world of cell-based molecules is vastly larger than the known chemical design space (Davies, 2011). De novo biomolecular production efforts have sought to capitalize on this space to generate new biofuels, commodity chemicals, and specialty chemicals (King et al., 2016). Beyond developing molecules with superior properties, biosustainable production of these molecules could contribute to a substantial reduction in carbon emissions, which is needed to avoid potentially devastating climate change (Matthews et al., 2009). Generally, biosynthesis of unnatural molecules often relies on broad substrate ranges (Rodriguez et al., 2014) and promiscuous activity in enzymes (Khersonsky et al., 2006). While major advances have been made in protein engineering, redesigning proteins to generate novel bioactivity and achieve new products remains a major challenge (Kumar et al., 2018).

Polyketide synthases synthesize an astonishing diversity of natural products including, but not limited to, anticancer, antimicrobial, and immunomodulating compounds (reviewed by (Robbins et al., 2016) ). Assembly-line, modular polyketide synthases (PKSs), a subset of Type 
I PKSs, are often linked in a collinear fashion, creating a design space that could be rationally reprogrammed to produce many valuable biomolecules (Cai and Zhang, 2018; Yuzawa et al., 2018b; Zargar et al., 2017, 2018). Each module's cycle begins with a Claisen-like condensation reaction between the growing chain on the ketosynthase (KS) domain and a malonyl-CoA analog on the acyl carrier protein (ACP) that was loaded by the acyltransferase domain (AT) (Figure 1A). Unlike fatty acid synthases that exclusively incorporate malonyl-CoA, AT domains of Type-I PKSs select a wide variety of extender units, greatly expanding the biological design space. After chain extension, the molecule's carbonyl reduction state is determined by the reductive domains present within a module, namely the ketoreductase $(\mathrm{KR})$, dehydratase $(\mathrm{DH})$, and enoylreductase (ER), which generate the $\beta$-hydroxyl, a- $\beta$ alkene (typically trans), or saturated $\beta$-carbons respectively when progressively combined; PKSs can have variability in $\beta$ carbon reduction, which is a major source of polyketide diversity and another attractive feature for molecular design. Finally, a thioesterase (TE) domain typically releases the final product from the megasynthase via hydrolysis or cyclization. While these biomolecular pathways have most often been engineered to fine-tune potential drug candidates, combinatorial biosynthesis could be implemented to generate molecules with simple scaffolds, such as biofuels and industrial chemicals (Cai and Zhang, 2018). While combinatorial biosynthesis of PKSs through domain modification, module swaps, and other techniques have made major progress in drug development (Hertweck, 2015; Weissman, 2016; Wong and Khosla, 2012), de novo biomolecular production is still a nascent field, and there have been no examples of multimodular PKS redesign to produce valuable biochemicals. 
A)

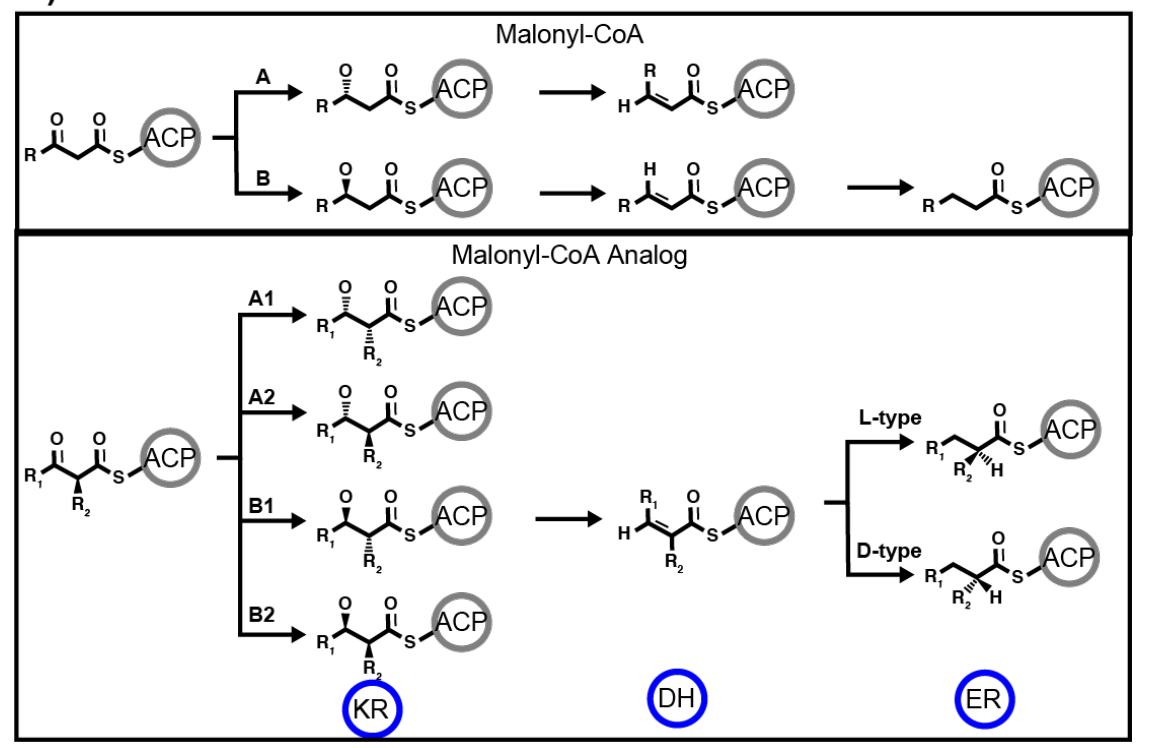

B)

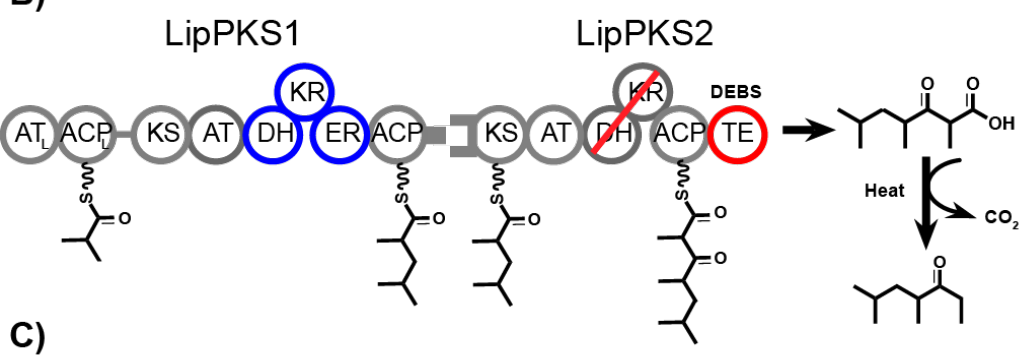

LipPKS1

LipPKS2

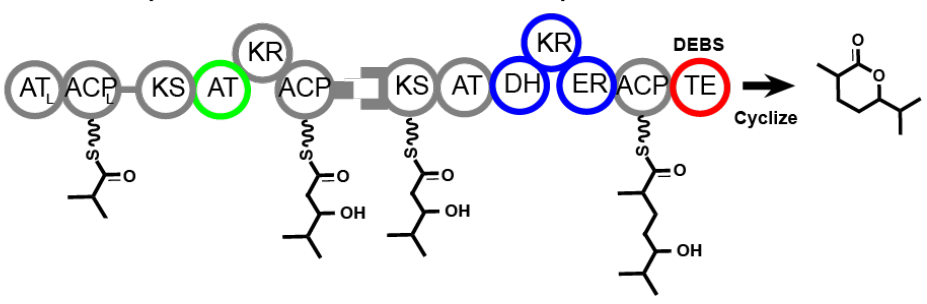

Figure 1. Schematic of PKS processing and engineering design in this study. A) PKS processing of each subtype of malonyl-CoA and malonyl-CoA analog extender units B) Lipomycin bimodular PKS design to produce ethyl ketones through a full reductive donor loop in LipPKS1 (blue circles), a KR mutant to abolish activity (red line), and a fused DEBS TE (red circle) C) Lipomycin bimodular PKS design to produce $\delta$-lactone through an AT-swap in LipPKS1 (green circle), a full reductive donor loop in LipPKS2 (blue circles), and a fused DEBS TE (red circle).

Previously, our group has engineered three major PKS elements in the first module of lipomycin: 1) an inserted TE to produce 3-hydroxy acids (Yuzawa et al., 2017a), 2) a KR knockout and AT domain swap to produce short chain ketones (Yuzawa et al., 2018a, 2017b), and 3) reductive loop $(\mathrm{RL})$ exchanges to produce saturated, short chain carboxylic acids (Zargar 
et al., 2019). The design space expands considerably with multiple module systems, and in this work, we build on our single module platform by combining multiple PKS manipulations (KR knockouts, reductive loop (RL) swaps, AT swaps, fused TE) in a biomodular system to produce novel biomolecules, namely biofuels and specialty chemicals.

\section{Materials and Methods}

\subsection{Cloning}

\section{Cloning of all constructs}

All clusters were expressed from the Streptomyces albus genome under control of the $\mathrm{GapDH}(\mathrm{El})$ promoter from Eggerthella lenta. Junction sites for reductive loop exchanges were determined by those reported by Hagen et al. through multiple sequence alignment with MUSCLE (Hagen et al. 2016; Edgar 2004). The plasmids along with their associated information have been deposited in the public version of JBEI registry (http://public-registry.jbei.org) and are physically available from the authors upon request https://public-registry.jbei.org/folders/557. Cloning of LipPKS1 with full reductive loop modules and native docking domain The $\phi$ C31-based Streptomyces integrase vectors were used as described by Phelan et al to integrate the LipPKS1 reductive loop swap modules (Phelan et al., 2017). The native docking domain sequences of LipPKS1 were codon optimized for E. coli and synthesized by Gen9 (since acquired by Ginkgo Bioworks). They were cloned through Golden Gate assembly into the LipPKS1 module with an inserted RL from NanA2 from Zargar et al (Zargar et al., 2019).

\section{Cloning of LipPKS1 with AT-swap and native docking domain}

The $\phi$ C31-based integrase vectors $\underline{\text { (Phelan et al., 2017) }}$ were used to integrate the AT-swapped LipPKS1 module into the $\phi \mathrm{C} 31$ site in the $S$. albus chromosome. The Lip1 native C-terminal docking domain sequences of LipPKS1 were cloned into the AT-swapped LipPKS1 module to 
replace the DEBS TE from Yuzawa et al. (Yuzawa et al., 2017b) through Golden Gate assembly.

\section{Cloning of LipPKS2 with KR knockout and fused DEBS thioesterase}

The VWB-based Streptomyces integrase vectors were used to integrate the LipPKS2 KR module (Phelan et al., 2017). The native LipPKS2 was codon optimized for $E$. coli with a single point mutation S1547A into the KR active site to mutate the catalytic serine to alanine, thereby abolishing KR activity, and synthesized by Gen9 (since acquired by Ginkgo Bioworks). The fused DEBS thioesterase domain was placed at the C-terminus of the ACP domain through Golden Gate assembly.

Cloning of LipPKS2 with full reductive loop modules and fused DEBS thioesterase

The VWB Streptomyces integrase vectors were used to integrate the LipPKS2 reductive loop modules cloned previously (Zargar et al., 2019).

\subsection{Genetic integration}

Conjugation of LipPKS1 constructs into S. albus J1074

Escherichia coli ET12567/pUZ8002 was transformed with LipPKS1 plasmids and selected for on LB agar containing kanamycin $(25 \mu \mathrm{g} / \mathrm{mL})$, chloramphenicol $(15 \mu \mathrm{g} / \mathrm{mL})$, and apramycin $(50$ $\mu \mathrm{g} / \mathrm{mL})$. A single colony was inoculated into $5 \mathrm{~mL}$ of LB containing kanamycin $(25 \mu \mathrm{g} / \mathrm{mL})$, chloramphenicol $(15 \mu \mathrm{g} / \mathrm{mL})$, and apramycin $(50 \mu \mathrm{g} / \mathrm{mL})$ at $37^{\circ} \mathrm{C}$. The overnight culture was used to seed $10 \mathrm{~mL}$ of LB containing the same antibiotics, and the new culture was grown at $37^{\circ} \mathrm{C}$ to an OD600 of 0.4-0.6. The E. coli cells were pelleted by centrifugation, washed twice with LB, and resuspended in $500 \mu \mathrm{L}$ of LB. Fresh S. albus J1074 spores were collected from a mannitol soy agar plate with $5 \mathrm{~mL}$ of $2 \mathrm{xYT}$ and incubated at $50^{\circ} \mathrm{C}$ for $10 \mathrm{~min}$. The spores $(500$ $\mu \mathrm{L})$ and the $E$. coli cells $(500 \mu \mathrm{L})$ were mixed, spread onto mannitol soy agar, and incubated at $30^{\circ} \mathrm{C}$ for 16 hours. $1 \mathrm{~mL}$ of both nalidixic acid $(20 \mu \mathrm{g} / \mathrm{mL})$ and apramycin $(40 \mu \mathrm{g} / \mathrm{mL})$ were added to the plate and allowed to dry. The plate was then incubated for $3-4$ days at $30^{\circ} \mathrm{C}$. 
A single colony was inoculated into TSB containing nalidixic acid $(25 \mu \mathrm{g} / \mathrm{mL})$ and apramycin $(25$ $\mu \mathrm{g} / \mathrm{mL}$ ). After 3-4 days, a $1 \mathrm{~mL}$ aliquot was taken for genomic isolation using the Maxwell kit (Promega, Cat\# AS1490, Madison WI). Successful integration was verified using qPCR. The remainder of the culture was spread onto a MS plate and incubated at $30{ }^{\circ} \mathrm{C}$ for $2-3$ days. The spores were collected from the plate with 3-4 $\mathrm{mL}$ of water and mixed with glycerol to prepare a $25 \%$ glycerol stock, which was stored at $-80^{\circ} \mathrm{C}$ for long-term storage.

\section{Conjugation of VWB integrase LipPKS2 constructs into LipPKS1-conjugated S. albus}

E. coli ET12567/pUZ8002 was transformed with LipPKS2 plasmids and selected for on LB agar containing kanamycin $(25 \mu \mathrm{g} / \mathrm{mL})$, chloramphenicol $(15 \mu \mathrm{g} / \mathrm{mL})$, and spectinomycin $(200 \mu \mathrm{g} / \mathrm{mL})$. A single colony was inoculated into $5 \mathrm{~mL}$ of LB containing kanamycin $(25 \mu \mathrm{g} / \mathrm{mL})$, chloramphenicol $(15 \mu \mathrm{g} / \mathrm{mL})$, and spectinomycin $(200 \mu \mathrm{g} / \mathrm{mL})$ at $37^{\circ} \mathrm{C}$. The overnight culture was used to seed $10 \mathrm{~mL}$ of LB containing the same antibiotics, and the new culture was grown at $37^{\circ} \mathrm{C}$ to an OD600 of $0.4-0.6$. The $E$. coli cells were pelleted by centrifugation, washed twice with LB, and resuspended in $500 \mu \mathrm{L}$ of LB. S. albus spores with an integrated LipPKS1, which were collected from a mannitol soy agar plate with $5 \mathrm{~mL}$ of $2 \mathrm{xYT}$ and incubated at $50^{\circ} \mathrm{C}$ for 10 min. The spores $(500 \mu \mathrm{L})$ and the $E$. coli cells $(500 \mu \mathrm{L})$ were mixed, spread onto mannitol soy agar, and incubated at $30^{\circ} \mathrm{C}$ for 16 hours. $1 \mathrm{~mL}$ of each nalidixic acid $(20 \mu \mathrm{g} / \mathrm{mL})$, apramycin $(40 \mu \mathrm{g} / \mathrm{mL})$, and spectinomycin $(400 \mu \mathrm{g} / \mathrm{mL})$ was added to the plate and allowed to dry. The plate was then incubated for $3-4$ days at $30^{\circ} \mathrm{C}$. A single colony was inoculated into TSB containing nalidixic acid $(25 \mu \mathrm{g} / \mathrm{mL})$, apramycin $(25 \mu \mathrm{g} / \mathrm{mL})$ and spectinomycin $(200 \mu \mathrm{g} / \mathrm{mL})$. After 3-4 days, a $1 \mathrm{~mL}$ aliquot was taken for genomic isolation through the Maxwell kit (Promega, Cat\# AS1490, Madison WI). Successful integration was verified through qPCR. The remainder of the culture was spread onto a MS plate and incubated at $30 \cdot \mathrm{C}$ for $2-3$ days. The spores were collected from the plate with 3-4 mL of water and mixed with glycerol to prepare a $25 \%$ glycerol stock, which was stored at $-80^{\circ} \mathrm{C}$ for long-term storage. 


\subsection{Production runs}

\section{S. albus production runs}

Engineered $S$. albus spores were grown in $12 \mathrm{~mL}$ of TSB medium containing nalidixic acid (50 $\mu \mathrm{g} / \mathrm{mL})$, apramycin $(50 \mu \mathrm{g} / \mathrm{mL})$ and spectinomycin $(200 \mu \mathrm{g} / \mathrm{mL})$ for $4-5$ days at $30^{\circ} \mathrm{C}$. Three $\mathrm{mL}$ of the overnight culture was used to seed $30 \mathrm{~mL}$ of $10 \%$ media 042 and $90 \%$ plant hydrolysate (Yuzawa et al., 2018a), supplemented with 2.4 grams/liter of valine and nalidixic acid (50 $\mu \mathrm{g} / \mathrm{mL}$ ), which was grown for 10 days at $30^{\circ} \mathrm{C}$. For production runs of lactones, an overlay of 4 $\mathrm{mL}$ of dodecane was added to retain the product.

\subsection{Sample preparation}

\section{Sample preparation for detection of acids}

To detect acid side products, $1 \mathrm{~mL}$ of each sample was centrifuged at $5000 \mathrm{~g}$ for 10 minutes and $200 \mu \mathrm{L}$ of the supernatant was removed. The supernatant was mixed with $200 \mu \mathrm{l}$ of $100 \mu \mathrm{M}$ hexanoic acid dissolved in methanol and filtered using Amicon Ultra Centrifugal filters, $3 \mathrm{KDa}$ Ultracel, 0.5 mL device (Millipore). $\beta$-hydroxy (3-hydroxy- 2,4-dimethylpentanoic acid) and saturated acids (2,4-dimethylpentanoic acid) were synthesized by Enamine (Cincinnati, USA) to greater than $95 \%$ purity.

\section{Sample preparation for bimodular production of triketide lactones}

$10 \mathrm{~mL}$ of each sample was mixed with $2 \mathrm{~mL}$ of diethyl ether in a 15-mL conical tube and vortexed for 5 minutes. Each conical tube was centrifuged at $5000 \mathrm{~g}$ for 10 minutes and $1 \mathrm{~mL}$ of ether was removed and placed into a $2-\mathrm{mL}$ flat bottom microcentrifuge tube. Air was gently blown over each sample in a chemical fume hood until dry. The extract was resuspended in 200 $\mu \mathrm{L}$ of methanol. 5-methyl-6-(propan-2-yl)oxan-2-one was synthesized by Enamine (Cincinnati, USA) to greater than $95 \%$ purity. 
One $\mathrm{mL}$ of each sample was harvested in a 1.7-mL microcentrifuge tube. To each tube, $300 \mu \mathrm{L}$ of ethyl acetate and $50 \mu \mathrm{L}$ of formic acid were added. All tubes were wrapped in paraffin and heated for 60 minutes at $80 \mathrm{C}$. Samples were then placed on ice for 5 minutes and vortexed for 5 minutes. Each sample was centrifuged for 2 minutes at $10,000 \mathrm{~g}$. One hundred microliters of ethyl acetate was removed from each sample and placed in a GC MS vial.

\subsection{Analytical chemistry}

\section{GC-MS detection of 4,6-dimethyl heptanone}

Electron ionization GC/MS analysis was performed on a G3950A-9000 GC (Agilent) using a J\&W HP-5ms Ultra Inert Intuvo GC column module (15 m length, 0.25 mm inner diameter, 0.25 $\mu \mathrm{m}$ film thickness). The GC was coupled to a mass selective detector (Agilent 5977B MSD) and an autosampler (Model 7693 Agilent). The GC oven was programmed at $60^{\circ} \mathrm{C}$ for 3 minutes, ramping at $10^{\circ} \mathrm{C} / \min$ until $120^{\circ} \mathrm{C}$, and then ramping at $200^{\circ} \mathrm{C} / \mathrm{min}$ to $300^{\circ} \mathrm{C}$; the injection port temperature was $250^{\circ} \mathrm{C}$. Using an authentic standard, we determined a single-ion method of detection collecting data at $\mathrm{m} / \mathrm{z}=57.00, \mathrm{~m} / \mathrm{z}=85.00, \mathrm{~m} / \mathrm{z}=142.00$.

\section{$\underline{\text { LC-MS detection of short chain acids }}$}

The LC-MS analysis was conducted on a Kinetex XB-C18 column (100-mm length, 3.0-mm internal diameter, and 2.6- $\mu$ m particle size; Phenomenex, Torrance, CA USA) using an Agilent Technologies 1200 Series HPLC system. The mobile phase for separating 2,4-

dimethylpentanoic acid and 2,4-dimethylpent-2-enoic acid was composed of $10 \mathrm{mM}$ ammonium acetate and $0.05 \%$ ammonium hydroxide in water (solvent $A$ ) and $10 \mathrm{mM}$ ammonium acetate and $0.05 \%$ ammonium hydroxide in methanol (solvent B). The mobile phase for separating 3hydroxy-2,4-dimethylpentanoic acid and 2,3-dimethyl-3-oxopentanoic acid was composed of $0.1 \%$ formic acid in water (solvent $\mathrm{A}$ ) and $0.1 \%$ formic acid in methanol (solvent $\mathrm{B}$ ). All acids were each separated via the following gradient: increased from 5 to $97.1 \%$ B in 6.5 min, held at 
$97.1 \% \mathrm{~B}$ for $1.3 \mathrm{~min}$, decreased from 97.1 to $5 \% \mathrm{~B}$ in $0.4 \mathrm{~min}$, and held at $5 \% \mathrm{~B}$ for an additional $2 \mathrm{~min}$. The flow rate was held at $0.42 \mathrm{ml} \cdot \min -1$ for $8.2 \mathrm{~min}$, and then increased from 0.42 to $0.65 \mathrm{ml} \cdot \min -1$ for an additional $2 \mathrm{~min}$. The total LC run time was $10.8 \mathrm{~min}$. Samples of $3 \mu \mathrm{l}$ were injected into the LC column. Acids were detected via $[\mathrm{M}-\mathrm{H}]-$ ions. Nitrogen gas was used as both the nebulizing and drying gas to facilitate the production of gas-phase ions. The drying and nebulizing gases were set to $11 \mathrm{~L} \cdot \min ^{-1}$ and $30 \mathrm{~L} \cdot$ bin-2- $^{-2}$, respectively, and a drying gas temperature of $330^{\circ} \mathrm{C}$ was used throughout. Atmospheric pressure chemical ionization was conducted in the positive-ion mode with capillary and fragmentor voltages of $3.5 \mathrm{kV}$ and $100 \mathrm{~V}$, respectively. The skimmer, OCT1 RF, and corona needle were set to $50 \mathrm{~V}, 170 \mathrm{~V}$, and $4 \mu \mathrm{A}$, respectively. The vaporizer was set to $350^{\circ} \mathrm{C}$. The analysis was performed using an $\mathrm{m} / \mathrm{z}$ range of 70 to 1100 . Data acquisition and processing were performed using MassHunter software (Agilent Technologies, United States).

\section{$\underline{\text { LC-MS detection of triketide lactones }}$}

LC separation of triketide lactones was conducted on a Kinetex XB-C18 reversed phase column (100 mm length, $3 \mathrm{~mm}$ internal diameter, $2.6 \mu \mathrm{m}$ particle size; Phenomenex, United States) using an Agilent 1200 Rapid Resolution LC system (Agilent Technologies, United States). The mobile phase was composed of water (solvent A) and methanol (solvent B). Lactones were each separated via the following gradient: increased from 30 to $90 \%$ B in 3.7 min, held at $94 \%$ B for $5.2 \mathrm{~min}$, decreased from 90 to $30 \% \mathrm{~B}$ in $0.33 \mathrm{~min}$, and held at $30 \% \mathrm{~B}$ for an additional $2.0 \mathrm{~min}$. The flow rate was held at $0.42 \mathrm{ml} \cdot \mathrm{min}^{-1}$ for $8.67 \mathrm{~min}$, increased from 0.42 to $0.60 \mathrm{ml} \cdot \mathrm{min}^{-1}$ in $0.33 \mathrm{~min}$, and held at $0.60 \mathrm{ml} \cdot \mathrm{min}^{-1}$ for an additional $2.0 \mathrm{~min}$. The total LC run time was $11.0 \mathrm{~min}$. The column compartment and autosampler temperatures were set to $50^{\circ} \mathrm{C}$ and $6^{\circ} \mathrm{C}$, respectively. Samples of $3 \mu \mathrm{L}$ were injected into the LC column. The Agilent 1200 Rapid Resolution LC system was coupled to an Agilent 6210 TOF (Agilent Technologies, United 
States). Nitrogen gas was used as both the nebulizing and drying gas to facilitate the production of gas-phase ions. The drying and nebulizing gases were set to $10 \mathrm{~L} \cdot \mathrm{min}^{-1}$ and $25 \mathrm{~L} \cdot \mathrm{bin}^{-2}$, respectively, and a drying gas temperature of $325^{\circ} \mathrm{C}$ was used throughout. Atmospheric pressure chemical ionization was conducted in the positive-ion mode with capillary and fragmentor voltages of $3.5 \mathrm{kV}$ and $100 \mathrm{~V}$, respectively. The skimmer, OCT1 RF, and corona needle were set to $50 \mathrm{~V}, 170 \mathrm{~V}$, and $4 \mu \mathrm{A}$, respectively. The vaporizer was set to $350^{\circ} \mathrm{C}$. The analysis was performed using an $\mathrm{m} / \mathrm{z}$ range of 70 to 1100 . Data acquisition and processing were performed using MassHunter software (Agilent Technologies, United States).

\section{$\underline{3.0 \text { Results }}$}

\section{$\underline{3.1 \text { Production of ethyl ketones }}$}

Short chain ketones (C3-C7) have been noted for their potential as gasoline additives because of their high octane numbers (McCormick et al., 2017). We recently tested their fuel combustion properties in a gasoline called CARBOB, a specially formulated Blendstock for Oxygenate Blending formula mandated by the state of California (Yuzawa et al., 2018a). While most ketones showed superior properties to the common biofuel butanol (octane numbers, energy density, boiling point, melting point, and flash point), methyl-branched C5 and C6 ketones had comparable fuel properties to isooctane. Longer chain ketones (above C7) were too expensive to synthesize for testing, but it is likely they are candidates as gasoline blending agents as well, and possibly possess combustion properties comparable or superior to traditional gasoline molecules. We therefore sought to produce longer chain ketones as an example of de novo production of biomolecules in a bimodular PKS system.

As illustrated in Figure 2A, we aimed to produce ethyl ketones through an RL exchange in LipPKS1 and an AT swap in LipPKS2. Previously, we had performed an RL exchange in LipPKS1 with a DEBS1-TE, and found a correlation between successful production of the 
desired product and the chemical similarity of the donor and recipient reductive loops with the most production through the chimera of LipPKS1 with an inserted donor loop form NanA2 (nanchangamycin, module 2) (Zargar et al., 2019). In the work presented here, we replaced the fused DEBS (6-deoxyerythronolide) thioesterase with the native docking domain of LipPKS1. For LipPKS2, we synthesized the codon-optimized gene with the native docking domain and a single point mutation at S1547A to mutate the catalytic serine to alanine in the KR domain. With the thioesterase of DEBS inserted following the ACP domain of LipPKS2, the programmed product of engineered Lip1 - Lip2 is a $\beta$-keto carboxylic acid, which, upon acidification and heat, is an ethyl ketone, 4,6-dimethyl heptanone.
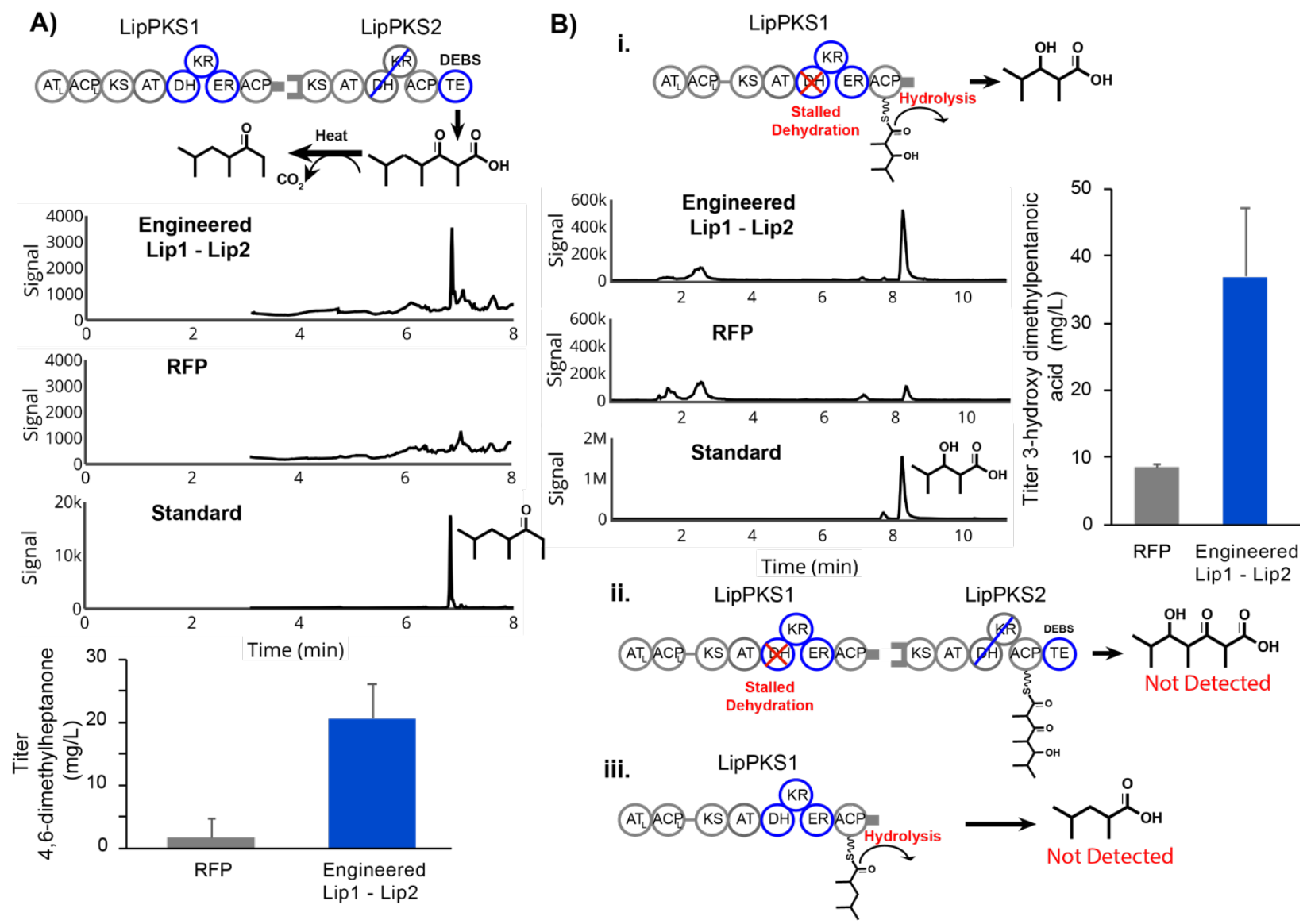

Figure 2. Production of ethyl ketones and side products in engineered Lip1 - Lip2 bimodular system. A) Schematic, MS chromatogram, and quantification of 4,6-dimethyl heptanone B) $i$. Schematic, MS chromatogram and quantification of the side product 3-hydroxy-2,4dimethylpentanoic acid due to incomplete reduction by LipPKS1 ii. Schematic of the side 
product of incomplete reduction in LipPKS1 processed and elongated by LipPKS2 iii. Schematic of the side product of complete reduction in LipPKS1 with premature hydrolysis

To produce the ethyl ketones, we conjugated the engineered Lip1 and Lip2 with the $\phi C 31$ or VWB integrases, respectively, into the genome of S. albus J1074. After 10-day production runs, we harvested the samples and measured titers of the final product and side products. We observe production of the desired product after heating and acidification with a titer of $20.6 \mathrm{mg} / \mathrm{L}$ (Figure 2A). While successful, this was a considerable drop in titer compared to the $165 \mathrm{mg} / \mathrm{L}$ of the saturated, carboxylic acid produced by the singular Lip1 extension module with NanA2 reductive loops and a fused TE. This loss of production is partially reflected in the amount of side products generated in the bimodular system. Previously, we found that the unimodular LipPKS with NanA2 reductive loops and a fused TE produced the DH-stalled product, 3-hydroxydimethylpentanoic acid (Zargar et al., 2019). We therefore suspected that incomplete $\beta$-carbon reduction by the engineered module 1 could cause premature hydrolysis of the product from the Lip1 ACP, resulting in production of the DH-stalled product, 3-hydroxy 2,4dimethylpentanoic acid, which was produced at a titer of $36.9 \mathrm{mg} / \mathrm{L}$, considerably higher than that produced by the negative control (Figure 2Bi). Previous studies on cis-AT PKS modules have shown that the elongating condensation reaction at the KS between the substrate and extender unit has higher selectivity than acylation of the KS by the substrate, a process generally known as gatekeeping (Watanabe et al., 2003; Wu et al., 2004). This may also explain the loss in titer compared to the engineered unimodular Lip1 $(\sim 165 \mathrm{mg} / \mathrm{L})$, as the stalled KS could reduce turnover. However, we did not observe this stalled $\beta$-hydroxy compound passed onto LipPKS2 and processed by the second module (Figure 2Bii). Importantly, the native Lip1 $\mathrm{KR}$ and donor NanA2 RL loop produce $\beta$-hydroxy compounds with different stereochemistries (A2 type compared to B1, Figure 1A). As it has been shown that KR domain exchanges generally retain native stereospecificity (Kao et al. 1998), we hypothesize that this difference in stereochemistry likely causes the downstream Lip2 KS to fail to elongate the stalled B1 type $\beta$ - 
hydroxy substrate of the engineered LipPKS1. On the other hand, we did not detect any of the fully reduced product, 2,4-dimethyl pentanoic acid, prematurely hydrolyzing as a saturated acid

(Figure 2Biii). This is in keeping with other studies that show KS domains have less promiscuity with bulkier substrates (Jenner, 2016).

\subsection{Production of $\delta$-lactones}

Over the past ten years, the market for genetically modified microbial production of fragrance aroma chemicals has grown. An important fragrant compound class is $\delta$-lactones (Gupta, 2015), and while not commercially used, 3-isopropyl-6-methyltetrahydropyranone has been synthesized previously as a potentially fragrant $\delta$-lactone (Plessis and Derrer, 2001). In a bimodular system with native AT domains, we previously engineered LipPKS1 and LipPKS2 to produce 3-isopropyl-4,6-dimethyltetrahydropyranone (Zargar et al., 2019), and in this work, we sought to incorporate AT-engineering to produce 3-isopropyl-6-methyltetrahydropyranone as an initial proof-of-concept production into this broad category of fragrant molecules.

The engineered production of this compound requires a combination of an AT swap in LipPKS1 and a RL swap in LipPKS2. For the AT swap in LipPKS1, we had previously analyzed AT domains and associated linkers to identify the boundaries for AT swaps while maintaining enzyme activity in LipPKS1 (Yuzawa et al., 2017b). We found that swapping the native methylmalonyl-CoA-selecting AT of LipPKS1 for the malonyl-CoA-selecting AT of the first module of the borreledin PKS (BorAT1), along with a fused DEBS TE, would result in the engineered unimodular system to produce 3-hydroxy carboxylic acids, which could be used as organic building blocks (Yuzawa et al., 2017b). Here, we replaced the fused TE with the native Lip1 docking domains in the BorAT1-swapped LipPKS1. For LipPKS2, we had previously introduced the NanA2 reducing loop, and when combined with the native LipPKS1, we had engineered production of 3-isopropyl-4,6-dimethyltetrahydropyranone (Zargar et al., 2019). This 
combination of LipPKS1 with the AT swap and LipPKS2 with an RL exchange should produce 3-isopropyl-6-methyltetrahydropyranone (Fig. 2A).

A)
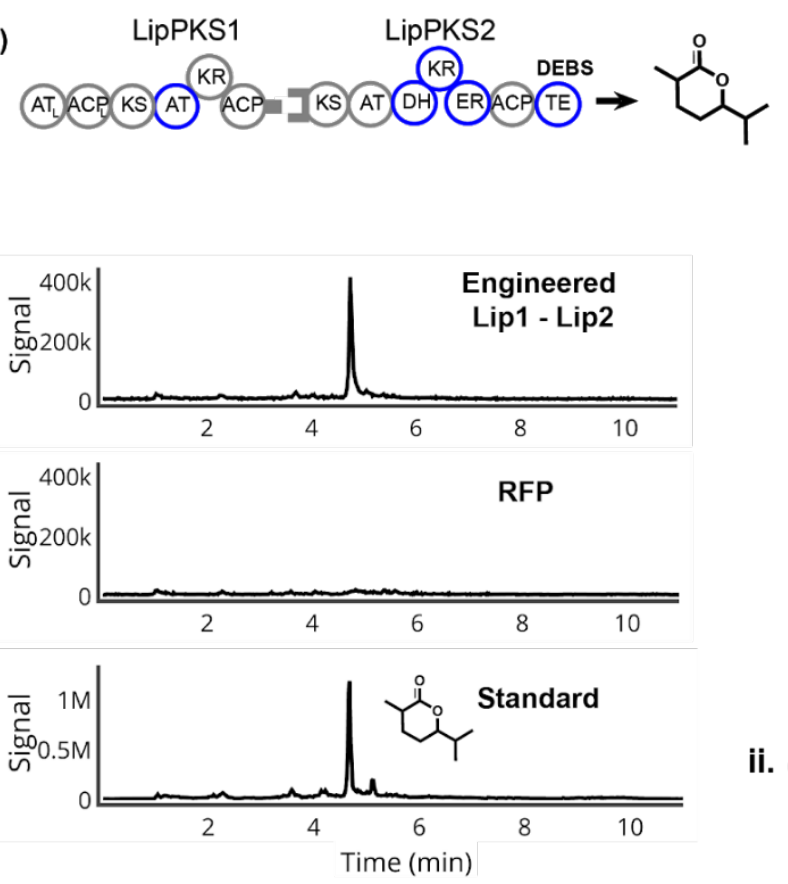

ii.
B) LipPKS1

i. $A T$ ACP- $\mathrm{KS}$ (AT)

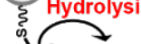

$\mathcal{f o H}^{\mathrm{oH}}$
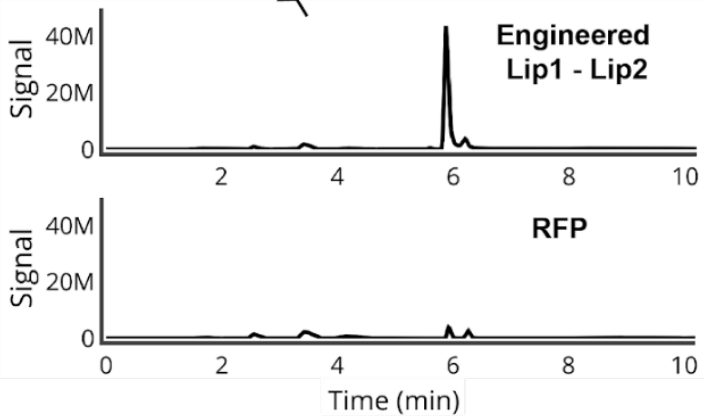

LipPKS1

LipPKS2
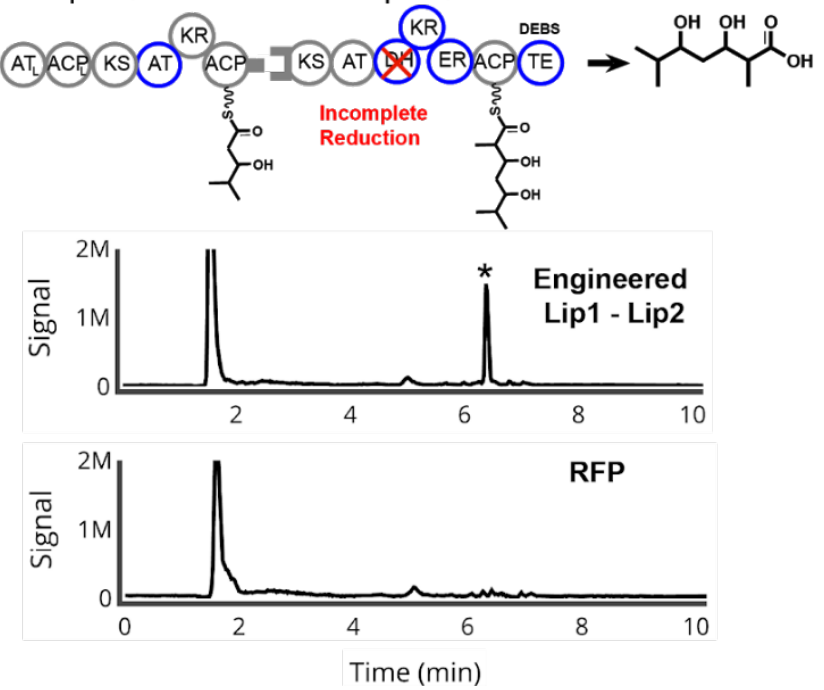

Figure 3. Production of $\delta$-lactone and side products in engineered Lip1 - Lip2 bimodular

system. A) Schematic, MS chromatogram, and quantification of 3-isopropyl-6-

methyltetrahydropyranone B) i. Schematic and MS chromatogram of the side product 3-

hydroxy-4-methylpentanoic acid due to premature hydrolysis of LipPKS1 ii. Schematic and MS chromatogram of the side product of incomplete reduction in LipPKS2 
As before, we integrated the genes encoding the engineered LipPKS1 and LipPKS2 into the genome S. albus J1074 at the i $\phi$ C31 and VWB phage attachment sites with $\phi$ C31 and VWB integrases, respectively. After 10 day production runs, we detected the programmed product with a titer of $40 \mu \mathrm{g} / \mathrm{L}$ (Figure $3 \mathrm{~A}$ ). We did not observe the product in the acid form, as 5dihydroxy 2,6-dimethyl heptanoic acid. This titer is considerably lower than the ethyl ketone titers from a similar bimodular PKS (20 mg/L). This could be caused by several factors including the non-native substrate presented to LipPKS2 by LipPKS1 with the malonyl-AT swap, disruption of the docking domain by the RL swap in LipPKS2, and lower turnover through the RL swap in LipPKS2. Once again, manipulation of the first module resulted in side products generated by premature hydrolysis at the LipPKS1. Premature hydrolysis of the AT swapped LipPKS1 would produce 3-hydroxy 4-methyl pentanoic acid, and we determined peaks of that mass in the culture (Figure 3Bi). As expected, we also observed production of 3,5-dihydroxy 2,6-dimethyl heptanoic acid, as the KR domain of the NanA2 reductive loop within LipPKS2 did not fully dehydrate the intermediate (Figure $\mathbf{3 B i i}$ ). The stalled $\mathrm{DH}$ product in the cyclized form, 5-hydroxy-3-isopropyl-6-methyltetrahydropyranone was not detected.

\section{Discussion}

While unaltered natural products and synthetic chemistry have been the basis of industrial molecules, de novo biomolecular designs allow an astonishing diversity of molecules that could have a transformational impact in many fields (Smanski et al., 2016). A biosynthetic platform based on PKSs represents a vast design space with an attractive programming basis. With the diversity of starter substrates $\left(\sim^{10^{2}}\right)$, malonyl-CoA analogs $\left(\sim 10^{1}\right)$, and stereochemistry arrangements $\left(\sim 10^{1}\right)$, a unimodular system alone can feasibly produce over 10,000 molecules and each subsequent module increases the number by two orders of magnitude. In the future, as our skills develop, we may be able to integrate our knowledge with other fields such as 'click' chemistry to obtain new capabilities (Le Feuvre and Scrutton 2018; Kalkreuter et al. 2019; Zhu 
et al. 2015). This would widely broaden both the scope of possible novel chemicals and our capacity to meet demands currently unable to be fulfilled.

In this study we've used a repertoire of AT domain swaps, mutagenesis, and reductive loop swaps with the goal of engineering a bimodular PKS while minimizing the disruption of protein-protein docking interactions. AT domain swaps enable the incorporation of rare extender units found in nature or even unnatural orthogonal extender units with click handles (Zhu et al. 2015) to expand the accessible chemical space. In our work, we performed an AT-swap to change the $\alpha$-substituent of our growing polyketide chain with the principles described before to successfully change the extender unit of LipPKS1 from methylmalonyl-CoA to malonyl-CoA (Yuzawa et al. 2017). The other major factor in polyketide diversity is the degree of $\beta$-carbon reduction, particularly for biofuels where oxygenation can directly affect physical attributes such as melting temperature, hygroscopicity, $\mathrm{H}: \mathrm{C}$ ratio, and vulnerability to oxidation (Wadumesthrige et al. 2009). We successfully employed a KR active site mutation in LipPKS2 to completely bypass reductive loop processing and ultimately yield ethyl ketones through the most conservative approach of active site mutagenesis. In contrast to the minimal disruption of a KR knockout, using reductive loop engineering principles recently described (Hagen et al. 2016) (Zargar et al. 2019), we were able to convert both LipPKS1 and LipPKS2 into fully reducing modules. This versatile bimodular platform paves the road for translating this strategy to a variety of PKSs with different loading modules and extension substrates to yield a diverse set of molecules. Additionally, a natural next step would involve extrapolating these engineering principles to PKS systems with more than 2 modules.

Failed production, reduced yield, and loss of product fidelity remain major challenges in PKS engineering. Online database tools such as ClusterCad (Eng et al., 2018) and SBSPKS $\underline{\text { (Khater et al. 2017) }}$ can facilitate PKS engineering, which can minimize the risk of failed production. A larger challenge is to increase fidelity and improve yield in multimodular systems, 
particularly as these systems are more likely to lose product fidelity as there is an added variable of intermodular interactions. Loss of product fidelity is not limited to domain and loop swaps as in this work; a recently engineered bimodular system successfully incorporated two non-native extender units through AT mutagenesis, but also produced unexpected side products through gatekeeping (Kalkreuter et al., 2019). Targeted mutagenesis of the ketosynthase can relieve the gatekeeping mechanisms, but the resulting activity is promiscuous, leading to loss of product fidelity (Jenner et al., 2015). Increased knowledge of PKS structure and function as well as machine learning algorithms on large datasets may inform future PKS designs to maximize product fidelity. Lastly, while improvements have been made in fermentation engineering to increase yield in hosts such as S. albus, strain engineering is still required to translate laboratory scale fermentation to industrial levels.

Previously, we have engineered the first module of lipomycin to make an array of molecules through AT swaps, RL swaps, KR knockouts, and a fused TE. Here, we have shown that we can leverage the knowledge we gained in unimodular systems to make multiple engineering manipulations to bimodular systems to produce novel biomolecules. While this is a step towards realizing the goals of capturing the biochemical design space, the unexpected metabolites produced through loss product fidelity highlight the need for a multi-factorial approach towards engineering these systems. Nonetheless, we successfully generated novel biofuels and specialty chemicals in the host Streptomyces albus.

\section{Acknowledgements}

This work was funded by the DOE Joint BioEnergy Institute (http://www.jbei.org) supported by the U.S. Department of Energy, Office of Science, Office of Biological and Environmental Research, through contract DE-AC02-05CH11231 between Lawrence Berkeley National Laboratory and the U.S. Department of Energy, and the National Institute of Health Awards F32GM125179. 


\section{Competing Financial Interest}

J.D.K. has a financial interest in Amyris, Lygos, Demetrix, Napigen, Maple Bio, Berkeley

Brewing Sciences, Ansa Biotech and Apertor Labs.

\section{Bibliography}

Cai, W., Zhang, W., 2018. Engineering modular polyketide synthases for production of biofuels and industrial chemicals. Curr. Opin. Biotechnol. 50, 32-38. doi:10.1016/j.copbio.2017.08.017

Davies, J., 2011. How to discover new antibiotics: harvesting the parvome. Curr. Opin. Chem. Biol. 15, 5-10. doi:10.1016/j.cbpa.2010.11.001

Eng, C.H., Backman, T.W.H., Bailey, C.B., Magnan, C., García Martín, H., Katz, L., Baldi, P., Keasling, J.D., 2018. ClusterCAD: a computational platform for type I modular polyketide synthase design. Nucleic Acids Res. 46, D509-D515. doi:10.1093/nar/gkx893

Gupta, C., 2015. A biotechnological approach to microbial based perfumes and flavours. JMEN 2. doi:10.15406/jmen.2015.02.00034

Hertweck, C., 2015. Decoding and reprogramming complex polyketide assembly lines: prospects for synthetic biology. Trends Biochem. Sci. 40, 189-199. doi:10.1016/j.tibs.2015.02.001

Jenner, M., 2016. Substrate Specificity of Ketosynthase Domains Part III: Elongation-Based Substrate Specificity, in: Using Mass Spectrometry for Biochemical Studies on Enzymatic Domains from Polyketide Synthases, Springer Theses. Springer International Publishing, Cham, pp. 131-154. doi:10.1007/978-3-319-32723-5_6

Jenner, M., Afonso, J.P., Bailey, H.R., Frank, S., Kampa, A., Piel, J., Oldham, N.J., 2015. Acyl-chain elongation drives ketosynthase substrate selectivity in trans-acyltransferase polyketide synthases. Angew Chem Int Ed Engl 54, 1817-1821. doi:10.1002/anie.201410219

Kalkreuter, E., CroweTipton, J.M., Lowell, A.N., Sherman, D.H., Williams, G.J., 2019. Engineering the Substrate Specificity of a Modular Polyketide Synthase for Installation of Consecutive NonNatural Extender Units. J. Am. Chem. Soc. 141, 1961-1969. doi:10.1021/jacs.8b10521

Khersonsky, O., Roodveldt, C., Tawfik, D.S., 2006. Enzyme promiscuity: evolutionary and mechanistic aspects. Curr. Opin. Chem. Biol. 10, 498-508. doi:10.1016/j.cbpa.2006.08.011

King, J.R., Edgar, S., Qiao, K., Stephanopoulos, G., 2016. Accessing Nature's diversity through metabolic engineering and synthetic biology. [version 1; peer review: 2 approved]. F1000Res. 5. doi:10.12688/f1000research.7311.1

Kumar, A., Wang, L., Ng, C.Y., Maranas, C.D., 2018. Pathway design using de novo steps through uncharted biochemical spaces. Nat. Commun. 9, 184. doi:10.1038/s41467-017-02362-x

Le Feuvre, R.A., Scrutton, N.S., 2018. A living foundry for Synthetic Biological Materials: A synthetic biology roadmap to new advanced materials. Synthetic and Systems Biotechnology 3, 105-112. doi:10.1016/j.synbio.2018.04.002

Matthews, H.D., Gillett, N.P., Stott, P.A., Zickfeld, K., 2009. The proportionality of global warming to cumulative carbon emissions. Nature 459, 829-832. doi:10.1038/nature08047

McCormick, R.L., Fioroni, G., Fouts, L., Christensen, E., Yanowitz, J., Polikarpov, E., Albrecht, K., Gaspar, D.J., Gladden, J., George, A., 2017. Selection Criteria and Screening of Potential Biomass-Derived Streams as Fuel Blendstocks for Advanced Spark-Ignition Engines. SAE Int. J. Fuels Lubr. 10, 442-460. doi:10.4271/2017-01-0868

Phelan, R.M., Sachs, D., Petkiewicz, S.J., Barajas, J.F., Blake-Hedges, J.M., Thompson, M.G., Reider Apel, A., Rasor, B.J., Katz, L., Keasling, J.D., 2017. Development of Next Generation Synthetic Biology Tools for Use in Streptomyces venezuelae. ACS Synth. Biol. 6, 159-166. doi:10.1021/acssynbio.6b00202 
Plessis, C., Derrer, S., 2001. Novel photolactonisation from xanthenoic esters. Tetrahedron Lett. 42, 6519-6522. doi:10.1016/S0040-4039(01)01296-5

Robbins, T., Liu, Y.-C., Cane, D.E., Khosla, C., 2016. Structure and mechanism of assembly line polyketide synthases. Curr. Opin. Struct. Biol. 41, 10-18. doi:10.1016/j.sbi.2016.05.009

Rodriguez, G.M., Tashiro, Y., Atsumi, S., 2014. Expanding ester biosynthesis in Escherichia coli. Nat. Chem. Biol. 10, 259-265. doi:10.1038/nchembio.1476

Smanski, M.J., Zhou, H., Claesen, J., Shen, B., Fischbach, M.A., Voigt, C.A., 2016. Synthetic biology to access and expand nature's chemical diversity. Nat. Rev. Microbiol. 14, 135-149. doi:10.1038/nrmicro.2015.24

Watanabe, K., Wang, C.C.C., Boddy, C.N., Cane, D.E., Khosla, C., 2003. Understanding substrate specificity of polyketide synthase modules by generating hybrid multimodular synthases. J. Biol. Chem. 278, 42020-42026. doi:10.1074/jbc.M305339200

Weissman, K.J., 2016. Genetic engineering of modular PKSs: from combinatorial biosynthesis to synthetic biology. Nat. Prod. Rep. 33, 203-230. doi:10.1039/c5np00109a

Wong, F.T., Khosla, C., 2012. Combinatorial biosynthesis of polyketides--a perspective. Curr. Opin. Chem. Biol. 16, 117-123. doi:10.1016/j.cbpa.2012.01.018

Wu, J., Kinoshita, K., Khosla, C., Cane, D.E., 2004. Biochemical analysis of the substrate specificity of the beta-ketoacyl-acyl carrier protein synthase domain of module 2 of the erythromycin polyketide synthase. Biochemistry 43, 16301-16310. doi:10.1021/bi048147g

Yuzawa, S., Bailey, C.B., Fujii, T., Jocic, R., Barajas, J.F., Benites, V.T., Baidoo, E.E.K., Chen, Y., Petzold, C.J., Katz, L., Keasling, J.D., 2017a. Heterologous Gene Expression of N-Terminally Truncated Variants of LipPks1 Suggests a Functionally Critical Structural Motif in the N-terminus of Modular Polyketide Synthase. ACS Chem. Biol. 12, 2725-2729. doi:10.1021/acschembio.7b00714

Yuzawa, S., Deng, K., Wang, G., Baidoo, E.E.K., Northen, T.R., Adams, P.D., Katz, L., Keasling, J.D., 2017b. Comprehensive in Vitro Analysis of Acyltransferase Domain Exchanges in Modular Polyketide Synthases and Its Application for Short-Chain Ketone Production. ACS Synth. Biol. 6, 139-147. doi:10.1021/acssynbio.6b00176

Yuzawa, S., Mirsiaghi, M., Jocic, R., Fujii, T., Masson, F., Benites, V.T., Baidoo, E.E.K., Sundstrom, E., Tanjore, D., Pray, T.R., George, A., Davis, R.W., Gladden, J.M., Simmons, B.A., Katz, L., Keasling, J.D., 2018a. Short-chain ketone production by engineered polyketide synthases in Streptomyces albus. Nat. Commun. 9, 4569. doi:10.1038/s41467-018-07040-0

Yuzawa, S., Zargar, A., Pang, B., Katz, L., Keasling, J.D., 2018b. Commodity chemicals from engineered modular type I polyketide synthases. Meth. Enzymol. 608, 393-415. doi:10.1016/bs.mie.2018.04.027

Zargar, A., Bailey, C.B., Haushalter, R.W., Eiben, C.B., Katz, L., Keasling, J.D., 2017. Leveraging microbial biosynthetic pathways for the generation of "drop-in" biofuels. Curr. Opin. Biotechnol. 45, 156-163. doi:10.1016/j.copbio.2017.03.004

Zargar, A., Barajas, J.F., Lal, R., Keasling, J.D., 2018. Polyketide synthases as a platform for chemical product design. AIChE J. doi:10.1002/aic.16351

Zargar, A., Lal, R., Valencia, L., Wang, J., Backman, T., Cruz-Morales, P., Kothari, A., Werts, M., Wong, A., Bailey, C.B., Loubat, A., Liu, Y., Benites, V., Chang, S., Hernandez, A.C., Barajas, J.F., Thompson, M., Barcelos, C., Anayah, R., Garcia-Martin, H., Keasling, J.D., 2019. Chemoinformatic-guided engineering of polyketide synthases. BioRxiv. doi:10.1101/805671 